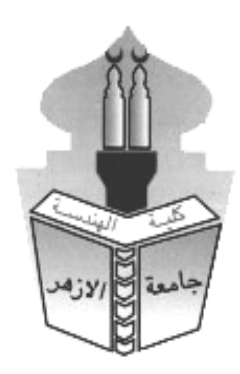

\title{
DEVELOPING OF THE EXISTING HOSPITALS TO COPE WITH EGYPTIAN HOSPITALS CODE
}

\author{
Sawsan Hussien Abd El Aleem*, Shaimaa Mohamed Kamel Mahmoud \\ and Yasser Mohamed Mansour \\ Department of Architecture- Faculty of Engineering- Ain Shams University, Cairo, Egypt \\ *Corresponding Author E-mail: sawsanelkotamy@ rocketmail.com
}

\begin{abstract}
Some existing hospitals suffer from the lack of medical services provided and the failure to follow the modern design standards for healthcare facilities. There is also a constant need to increase the number of hospitals and increase the medical services provided to cope with modern standards, so it is important to develop existing hospitals and utilize available resources.This research aims to shed light on the importance of developing existing hospitals, identifying different strategies for development and identifying the design considerations required when developing an existing hospital.Through the theoretical study of the main components of hospitals with a general specialty and the study of functional relationships between different elements and identify methods, strategies for development and studying the rules of renovation for existing Egyptian hospitals, and then do a analytical study for one of the hospitals where the development process was carried out and also the study of the reasons that led to resort to the development process.
\end{abstract}

The research also analyzed the strategies that were followed to develop the hospital and identify the positive results of the development process. And through this was reached the possibility of making some architectural changes to achieve design standards and meet the needs of the community.

KEYWORDS: Hospitals Renovation- Renovation Strategies- Existing Hospitals- Design Standards for Hospitals-Functional Renovation

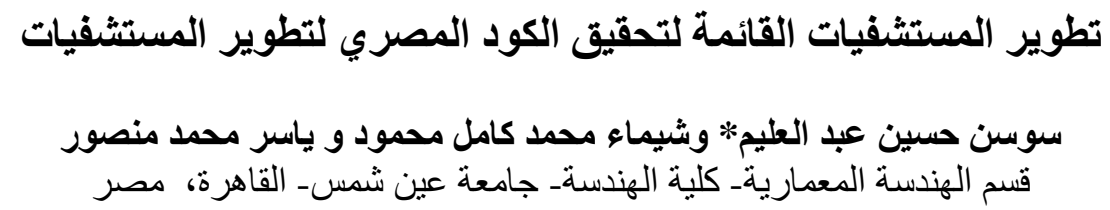

E-mail: sawsanelkotamy@ rocketmail.com : البريد الاليكتروني للباحث الرئيسية

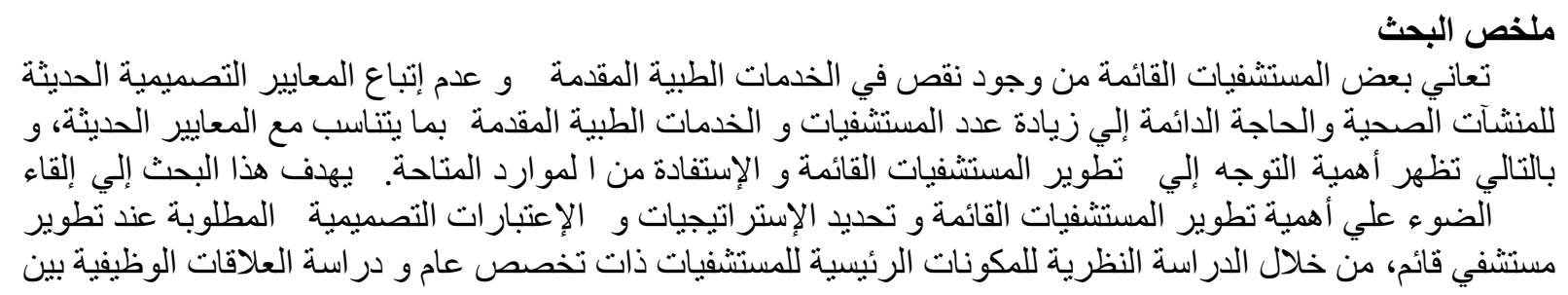


العناصر المختلفة و تحديد أساليب و إستر اتيجيات التطوير و دراسة الإشراطات التصميمية لتطوير المستشفيات المصرية

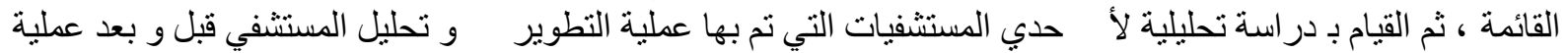

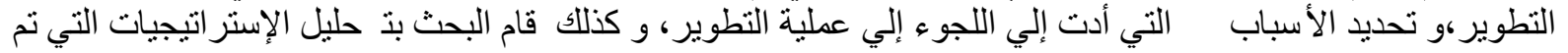

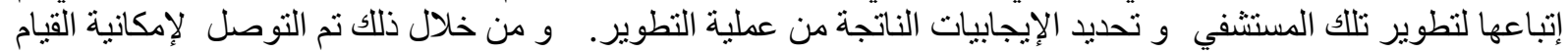
ببعض التغييرات المعمارية لتحقيق المعايير التصميمية و تحقيق حاجة المجنمع.

الكلمات المفتاحية : تطوير المستثفيات- إستراتيجيات التطوير- المستثفيات القائمة. المعايير التصميمية للمستثفياتـ التطوير الوظيفى : تطوير الميفيات

المقدمة

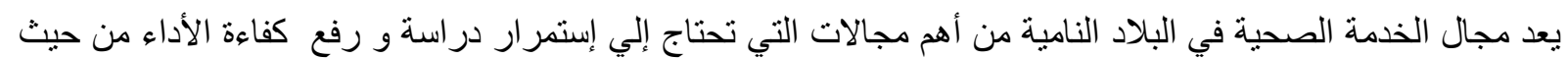

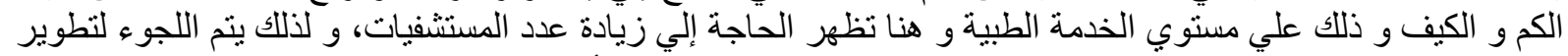

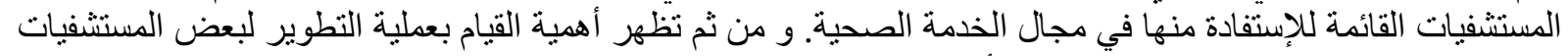

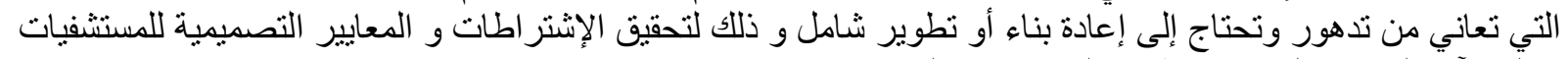

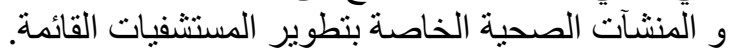

لذلك يهدف البحث دراسة إحدي المستشفيات التي تم بها التطوير لتحديد الإستر اتيجيات المتبعة في عملية التطوير و إلقاء

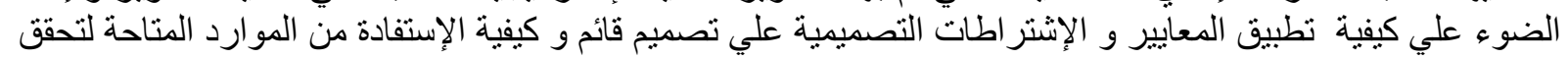
الخدمات الطبية الناقصة و تلبية إحتياجات المجتمع.

المثكلة البحثية

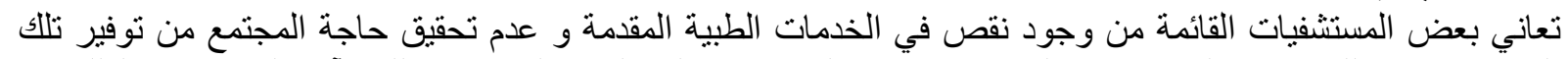

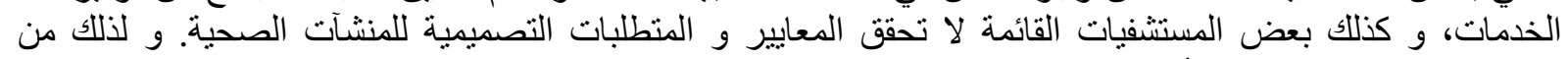

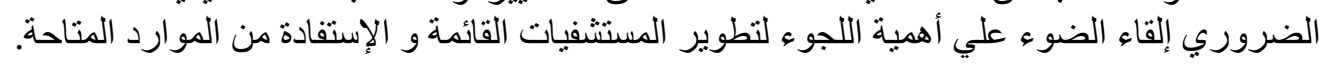

الاهدف من البحث

يهدف البحث إلي إلقاء الضوء الضو علي الإستراتيجيات المختلفة لتطوير المستتفيات القائمة و المنهجية المتبعة للقيام بعملية

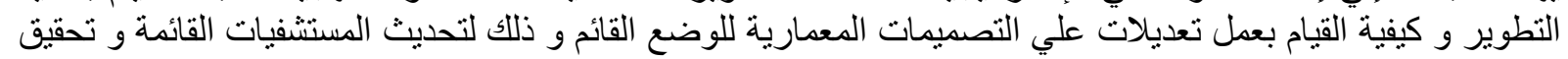

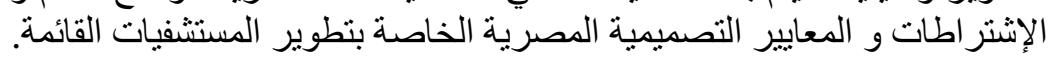

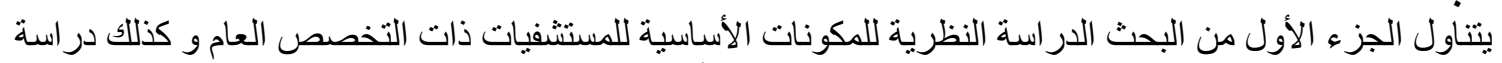

منهجية البحث

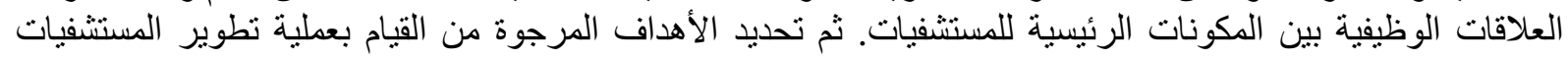

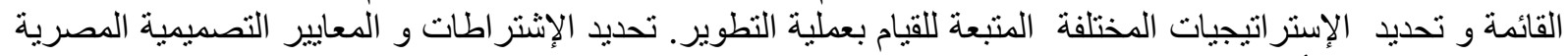
المطلوبة عند تجديد أو تطوير المستشفيات القيات القيائمة.

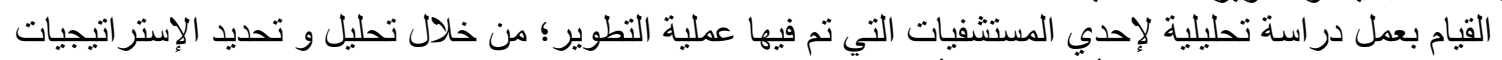

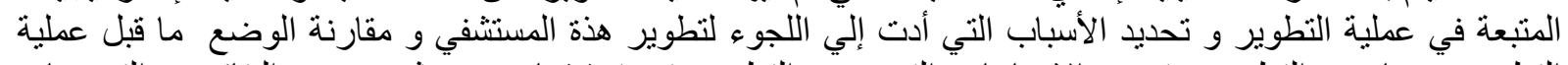

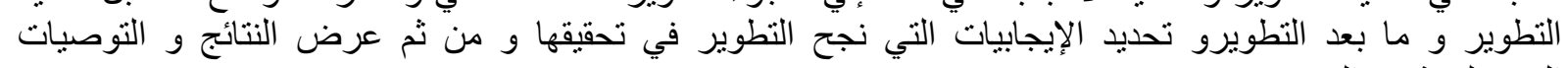

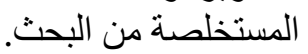

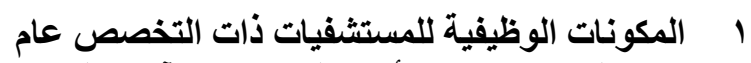

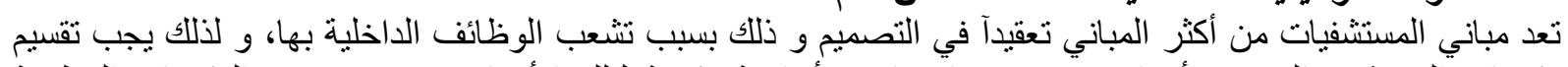

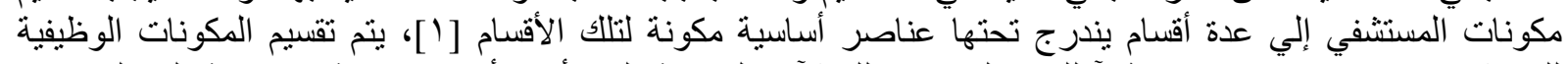

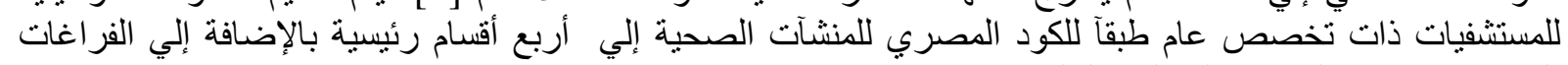

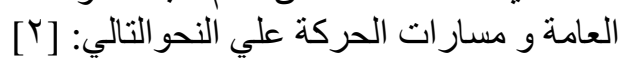

1 - الأقسام الداخلية ( الإقامة)

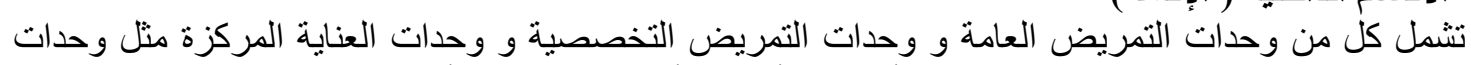

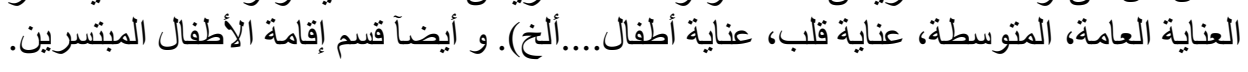




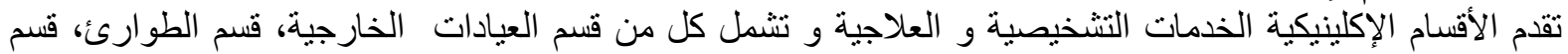

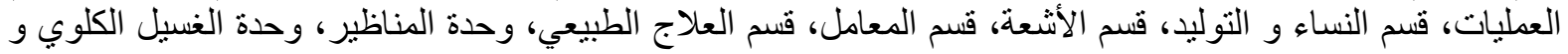

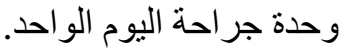

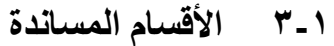

تشمل كل من الخدمات الطبية كقسم التعقيم المركزي و الصيدلية التية المركزية و المشرحة، و الخدمات الغير طبية

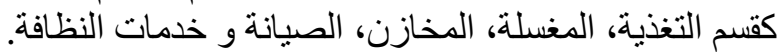

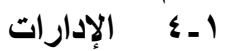

تتكون من قسم الإدارة الطبية ( سجلات طبية، تعليم طبي)، والإدارة الغير طبية ( إدارة مالية، علاقات عامة، شؤون عاملين...ألخ).

r العلاقات الوظيفية للعناصر المختلفة المكونة للمستثفيات العامة

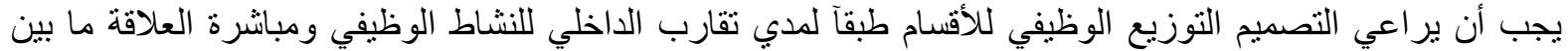

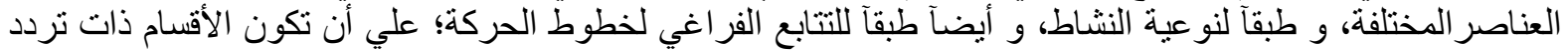

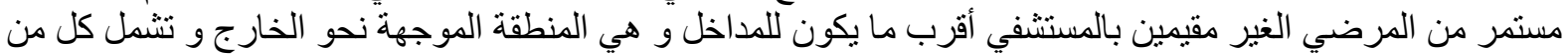

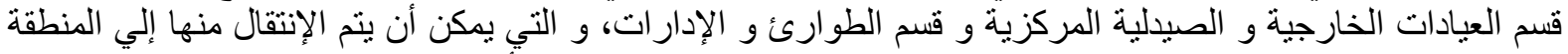

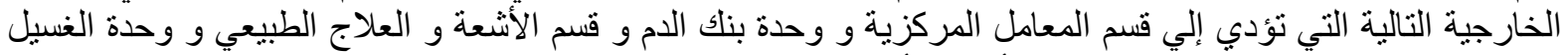

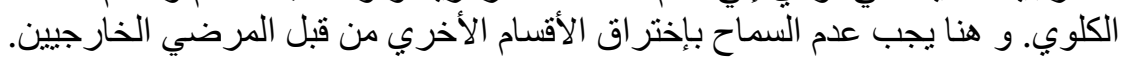

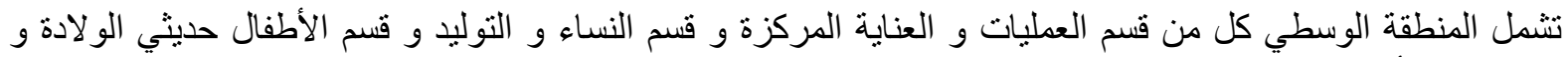

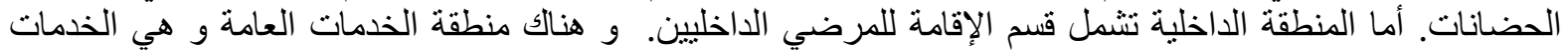

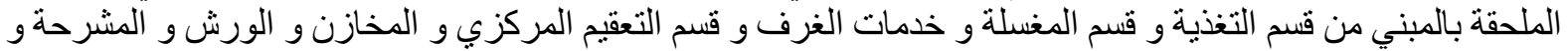

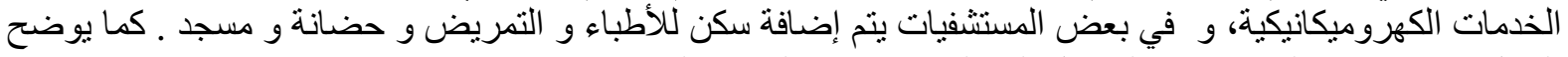

الثكل (1) عناصر المستشفي و المداخل المختلفة و خطوط الحركة الرئيسية بها. [ 1 ]

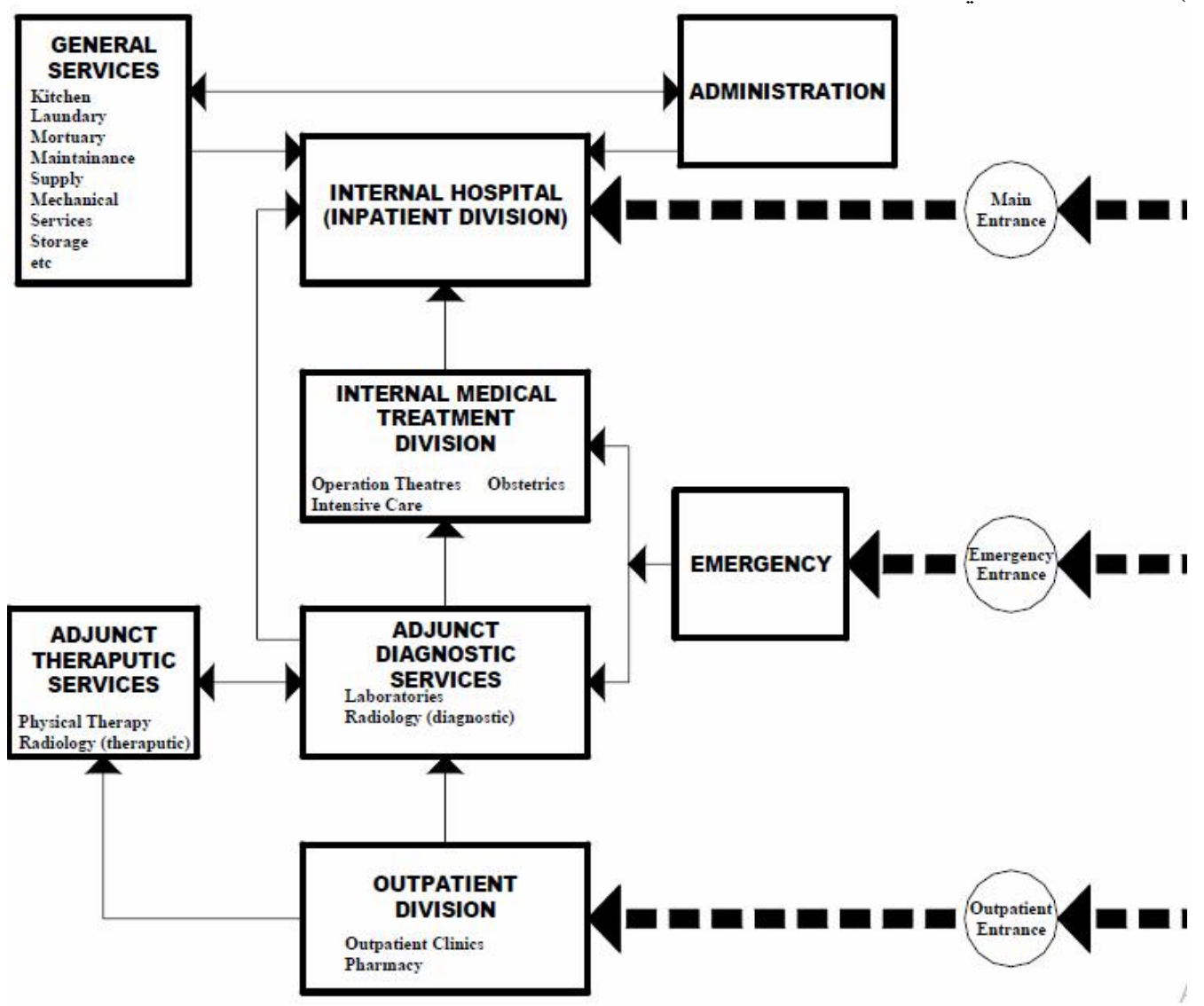

شكل ( (1): يوضح عناصر المستثفي و المداخل المختلفة و خطوط الحركة الرئيسية بها 


\section{المرجع: علي، هثام حسن، "محاضرات في تخطيطو تنسيق المستثفيات"، ص^}

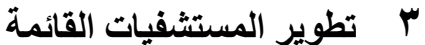

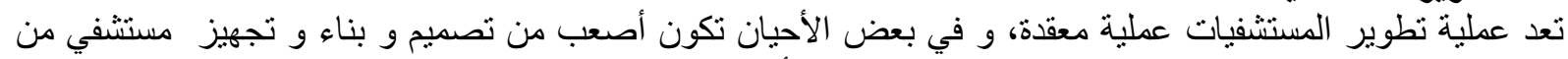

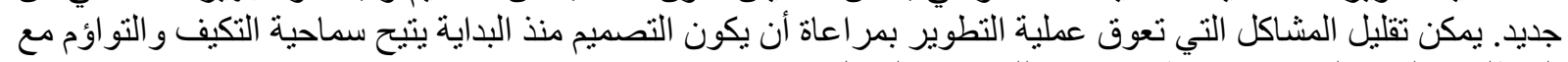
المنطلبات المستقبلية بحيث إعطاء إمكانية للتغيير و التبديل.

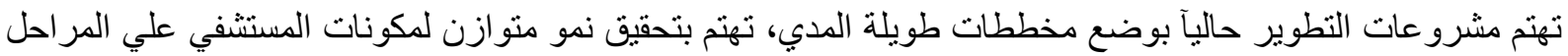

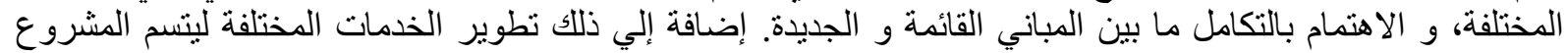

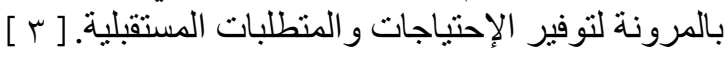

\section{؛ معني و مفهوم تطوير مباني المستثفيات}

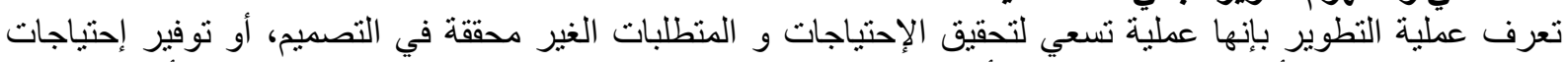

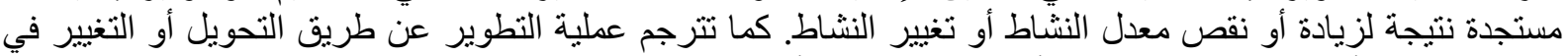

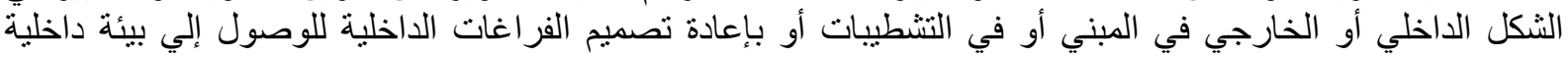

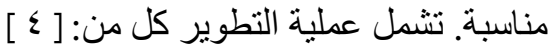
أ) التطوير داخلياً في المبني القائم ليتناسب مع الإحتياجات الجديدة. ب) إز الة مبني قائم تمامآ و ذللك لعدم تحقيق الإحتياجات المطلوبة أوطبقآ لمتطلبات السلامة الإنشائية، و في هذة الحالة

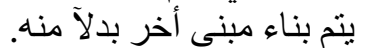
ت) إضافة مبني أو مباني أخري للمبني القائم لتوفير المنطلبات المستجدة و التي يصعب توفير ها في المبني القائم.

\section{ع ــ إن الهوف من عملية تطوير مباني المستثفيات}

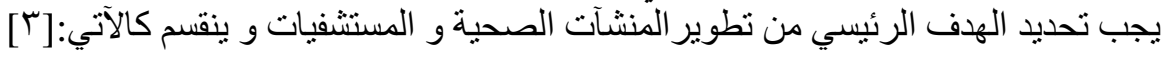

ـ ـ إن إستكمال العناصر الناقصة

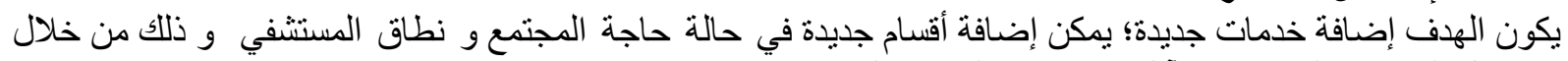
إستكمال العناصر الناقصة طبقآ لحاجة سكان المنطقة المحيطة.

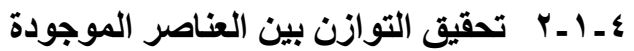
يتم التطوير في هذة الحالة عن طين طريق زيادة العياد حجم العنصر و آي من العناصر الناقصة لتحقيق التوازن، و ذلك في حالة وجود نقص في الفر اغات في قسم موجود بالفعل.

ـ ـ ا_ـ تحقيق زيادة كلية في حجم العناصر

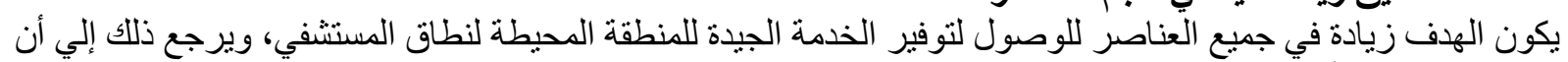
حجم المستشفي الأساسي غير كافي و لا مناسب لتوفير إحتياجات المنطقة المحيطة.

ـ ـ ا-ـ تحديث التجهيزات الفنية

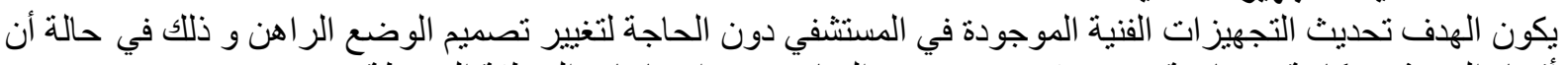
أقسام المستشفي كافية و مناسبة من حيث حجم و نسب العند العناصر مع إحتياجات المنطقة المحيطة.

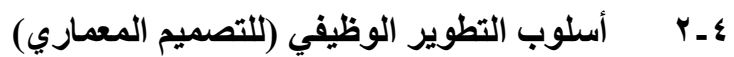

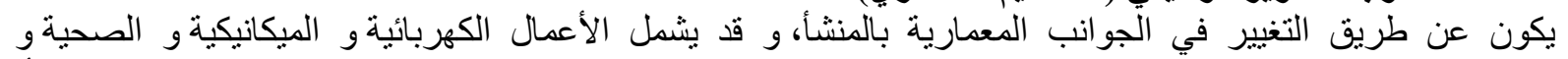

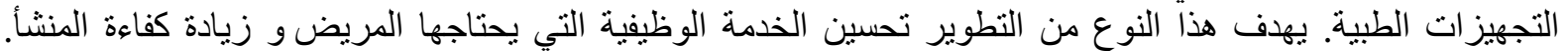
ويكون هذا النطوير عن طريق التغيير في العناصر، أو الإمتداد و النمو أو القيام بعملية الإز الذة و الإستبدال. [ب] 


\section{ـ ـ ـ ـ ا التطوير الوظيفي عن طريق التغيير}

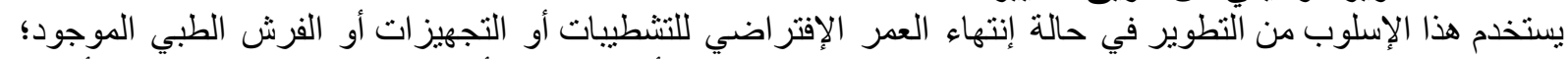

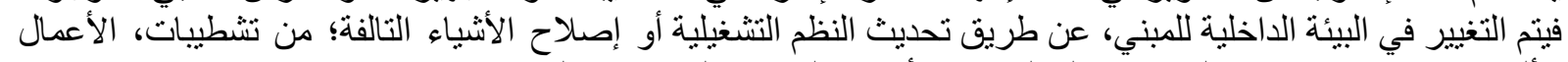

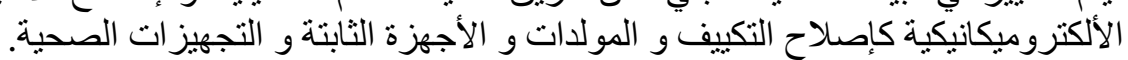

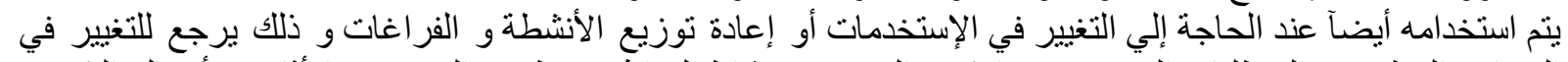

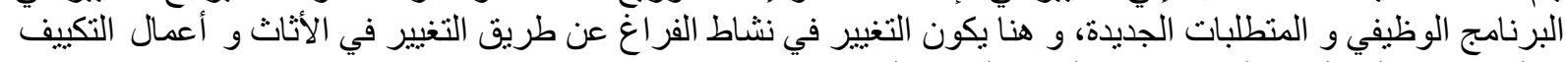

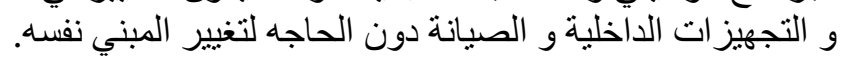

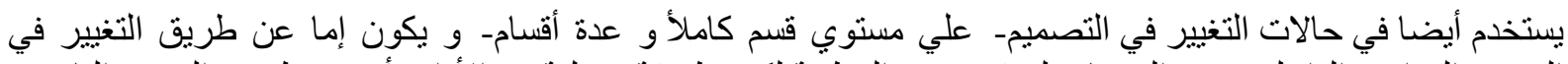

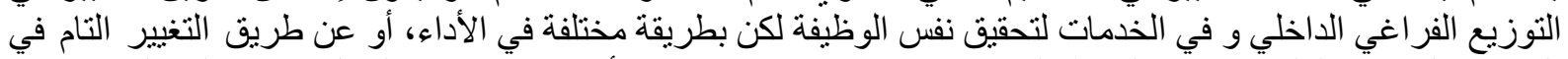

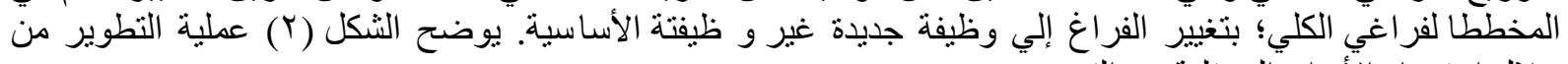
خلال إستخدام الأنواع "المختلفة من التغيير.

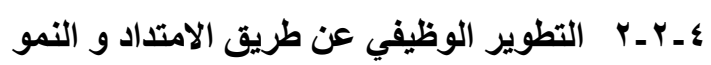

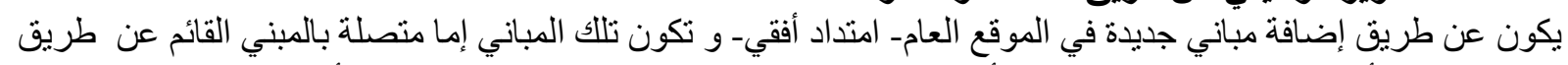

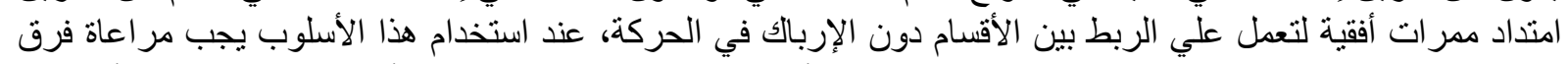

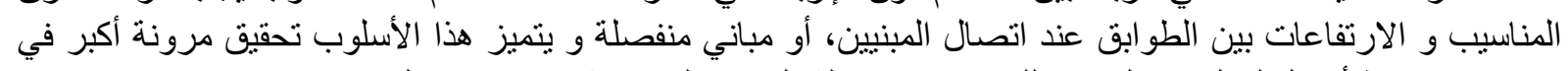

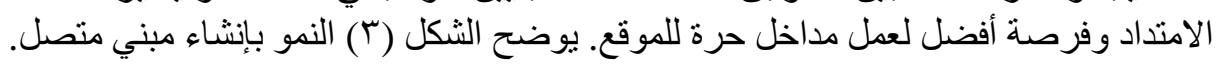

يمكن تحقيق الامتداد و النمو رأسيا؛ بإضافة أدوار أعلي أو أسفل المبني القائم. ولكن يجب مر اعاة تقرير السبامة السامة الإنشائية للمبني القائم و قابلية النظم الميكانيكية و الكهربائية للامتداد و الشتر اطات الموات المقع بالنسبة للارتفاعات و قو انين التنظيم. 

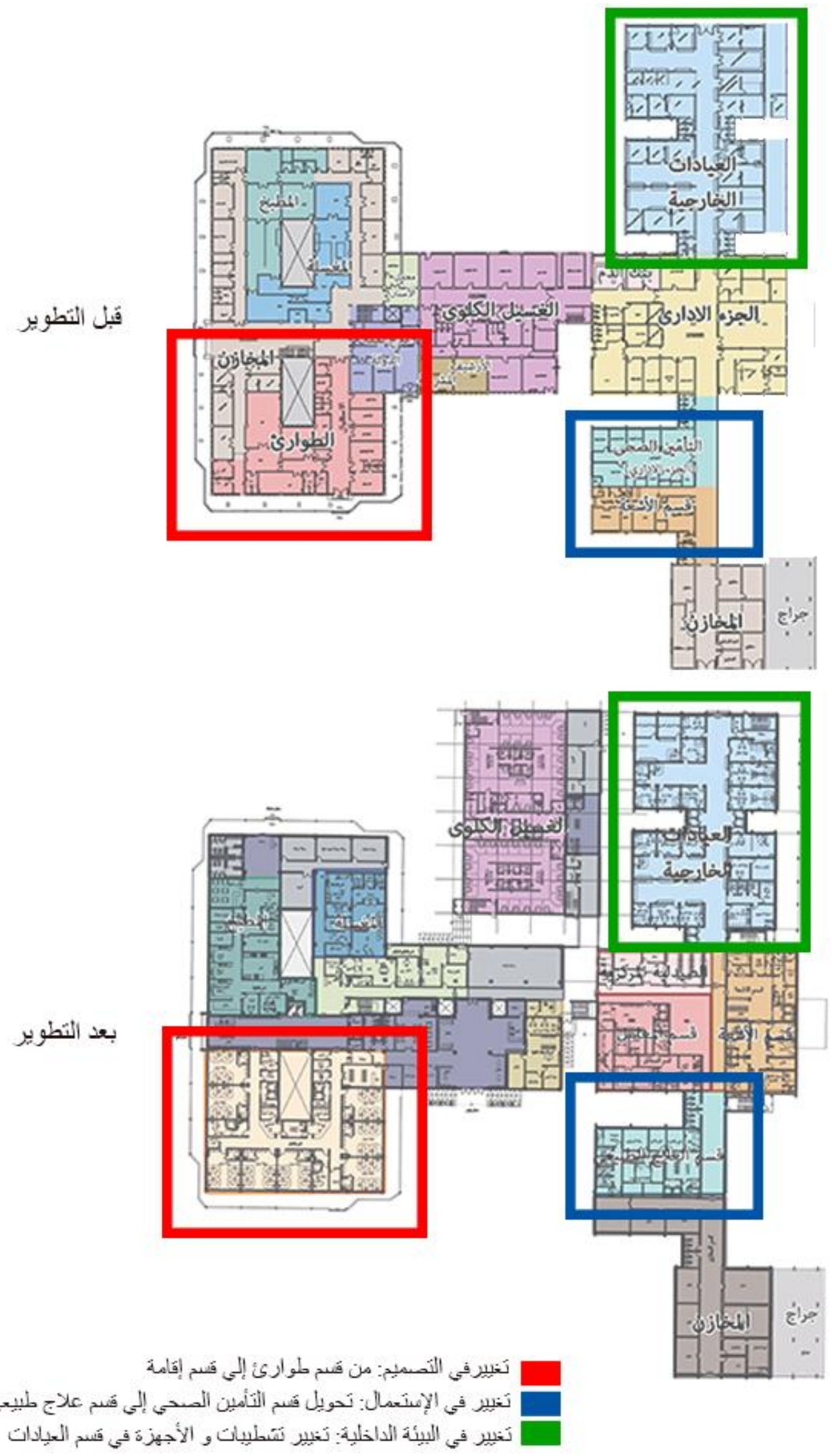

شكل (ץ): مسقط أفقي للاور الأرضي بمستثفي كفر شكر يوضح عملية التطوير من خلال إستخدام الأنواع المختلفة من التغيير المرجع: الباحث 


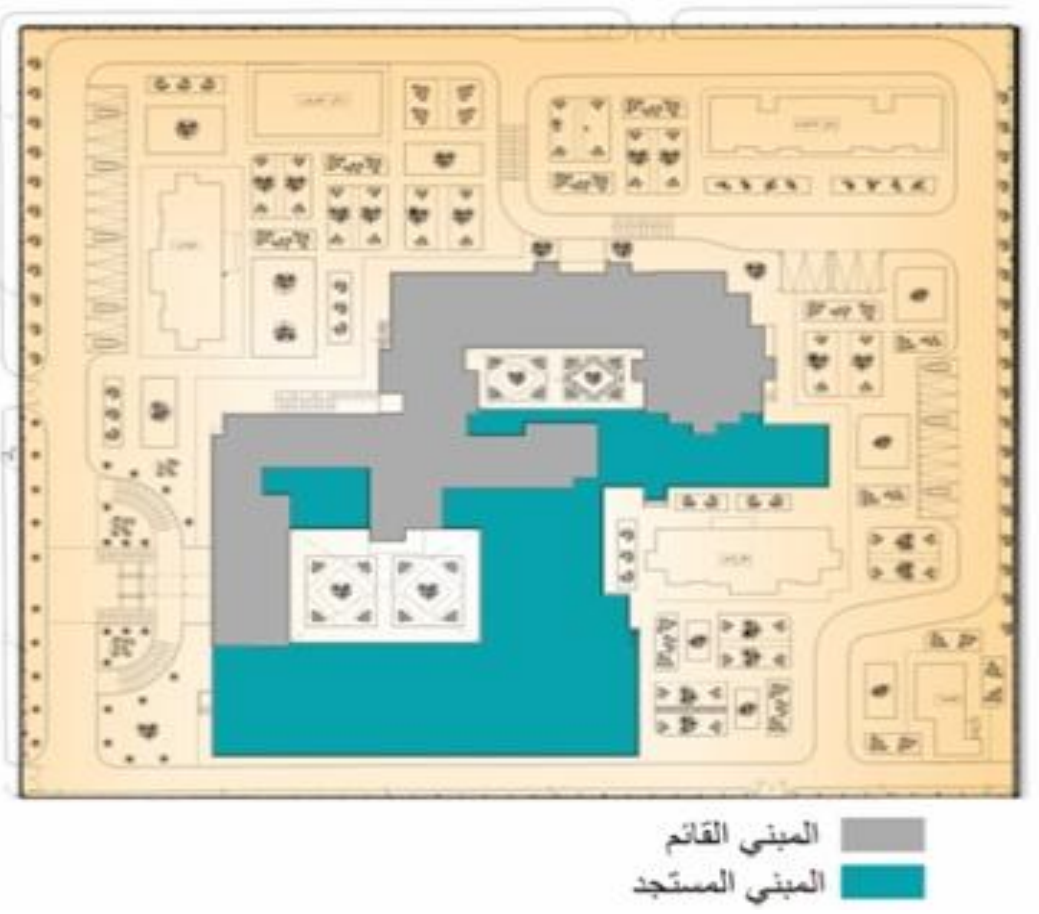

\section{شكل ("r): الموقع العام لمستثفي شلاتين المركزي يوضح النمو بإنثاء مبني متصل المرجع: الباحث}

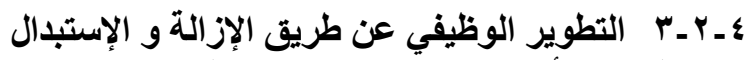

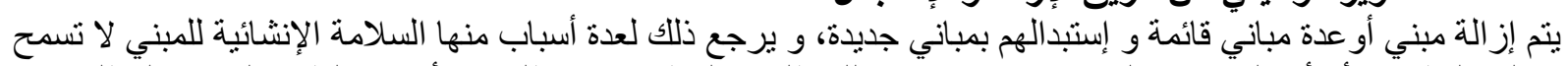

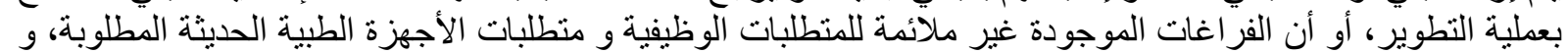

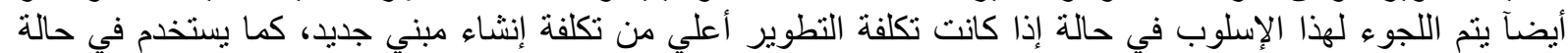

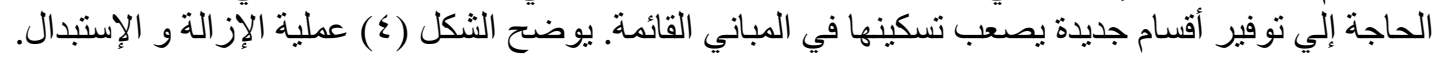

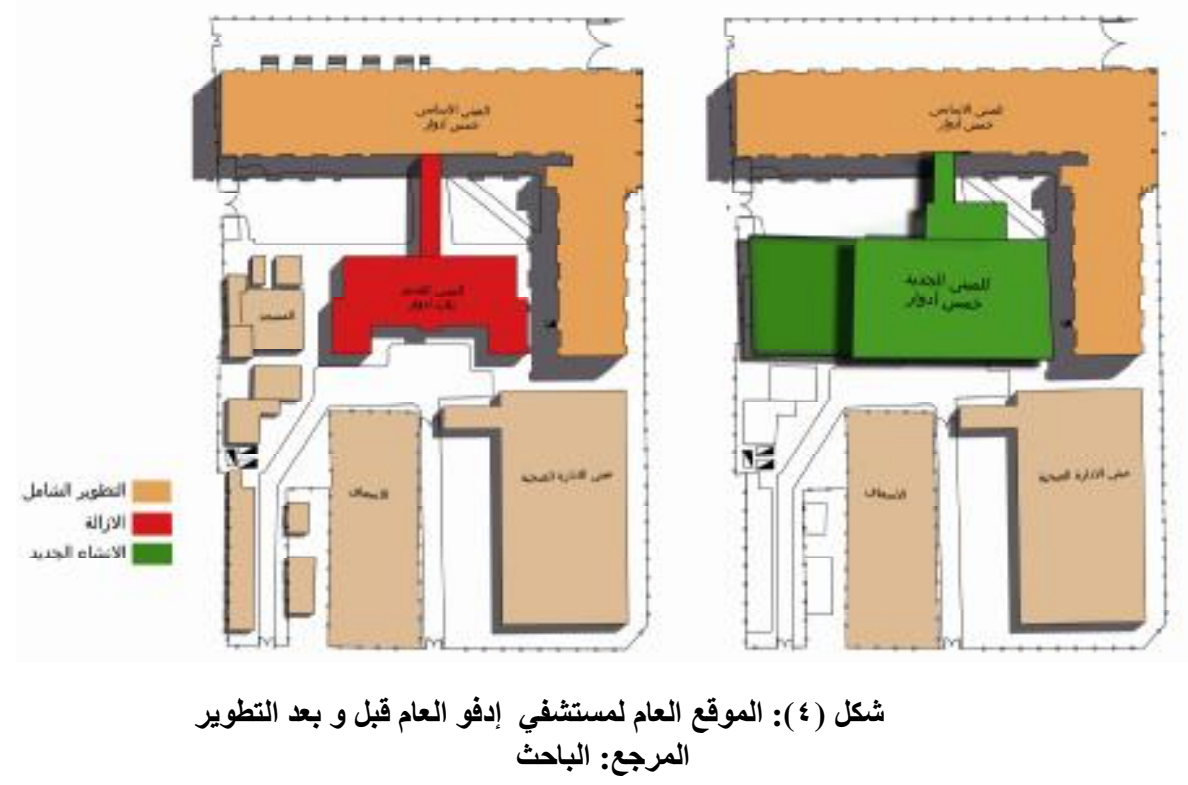


ه الاشتراطات و المعايير التصميمية المطلوبة عند تجديد أو تطوير المستشفيات القائمة

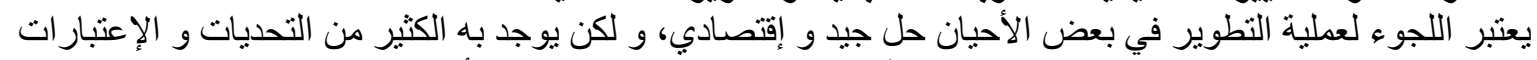

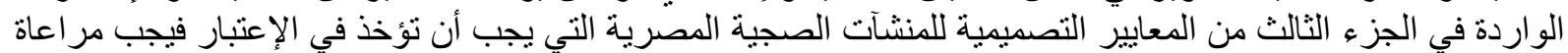
ا. في حالة إنشاء أقسام جديدة أو جناح ملحق بالمبني القائم يجب أن يتبع المعايير المصرية التصميمية للمستشفيات و التصو

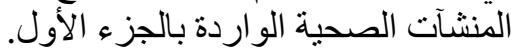

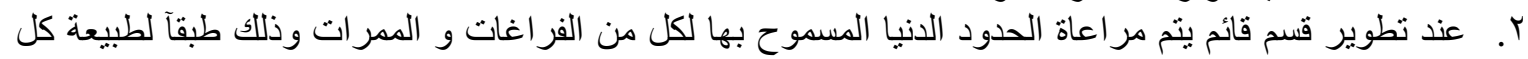

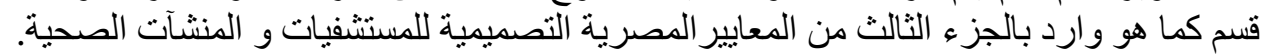

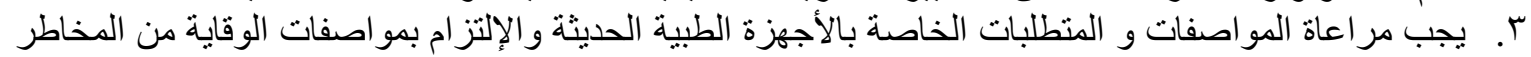
المختلفة كالمخاطر الإشعاعية و البيلوجية و الكيات الكيماوية.

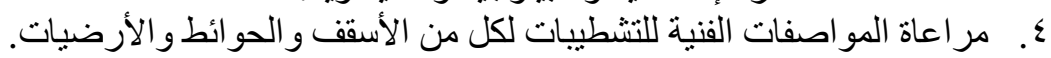

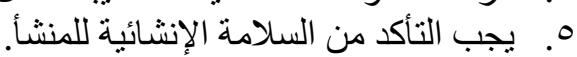

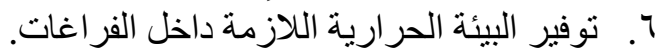

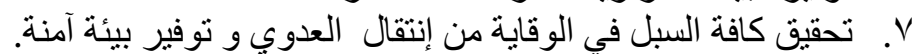

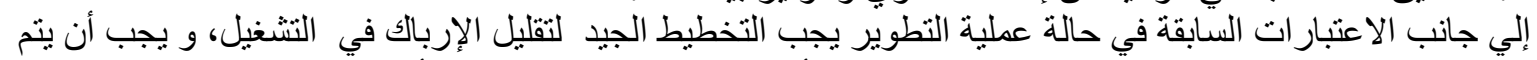

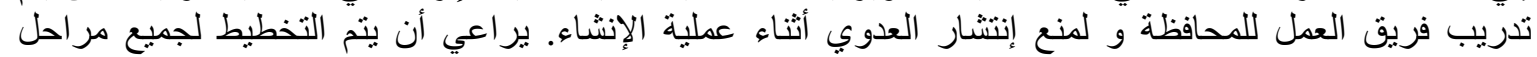

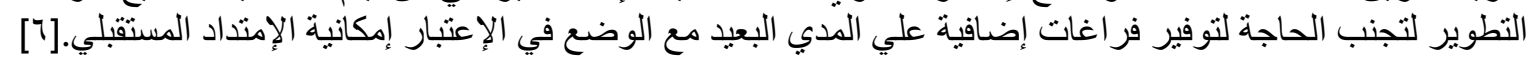

7

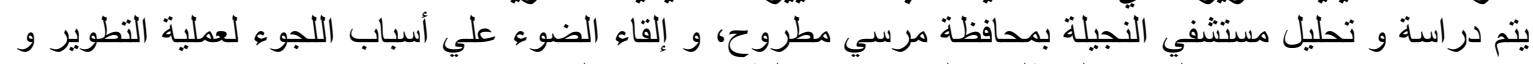
كيفية تحديث مستشفي قائم لتوفير المتطلبات التصميمية و الطبية و زيادة السعة.

؟ـ ا ـ تحليل المستشفي قبل عملية التطوير

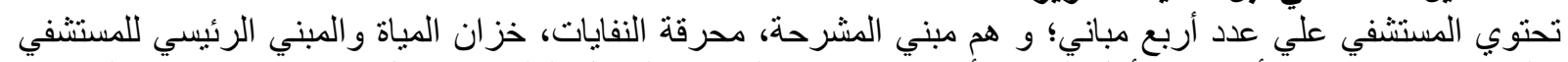

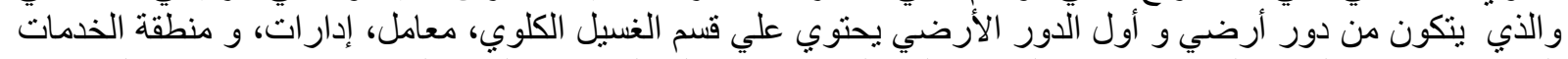

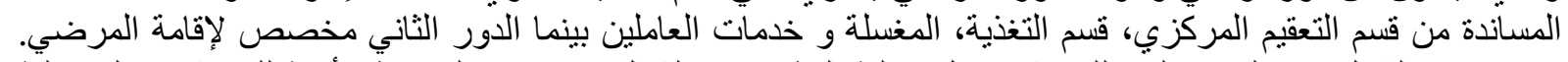

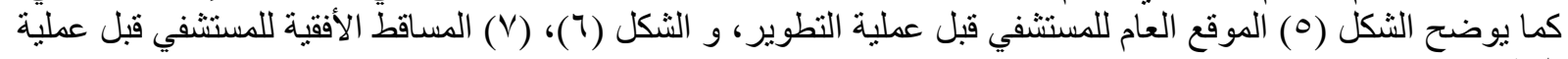
النطوير.

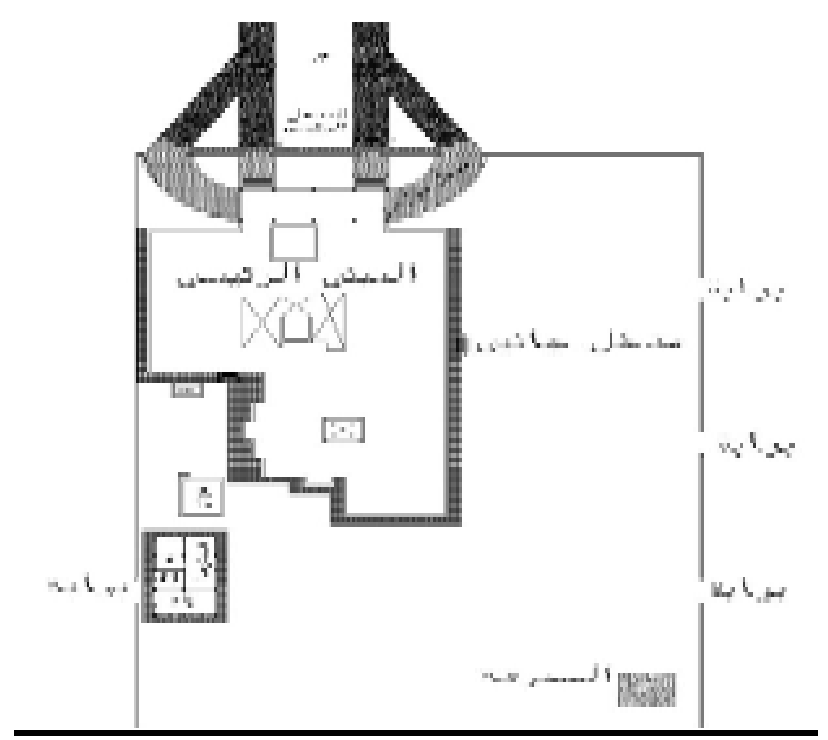

شكل (•): الموقع العام لمستثفي النجيلة قبل عملية التطوير 


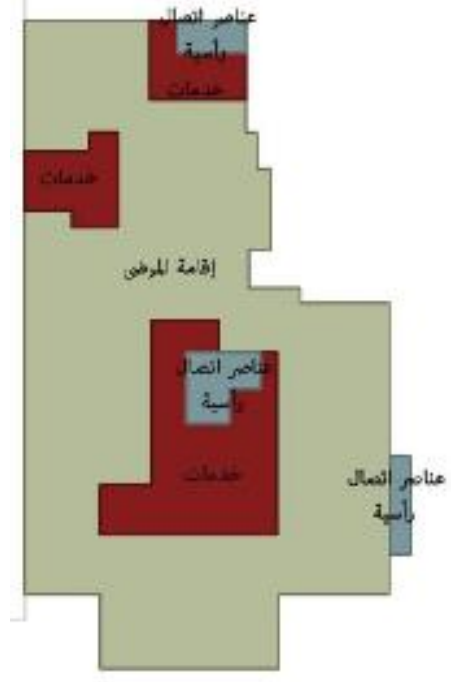

شكل (V): تحليل المسقط الأفقي للاور الأول قبل عملية التطوير

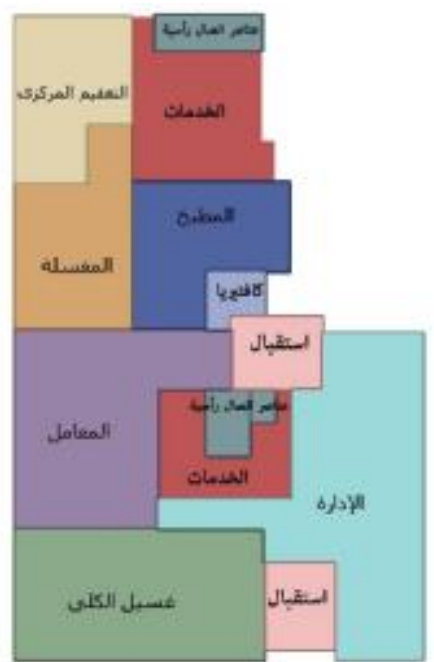

شكل (ף): تحليل المسقط الأفقي للاور الأرضي قبل عملية التطوير

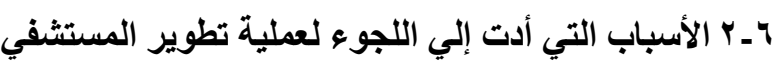

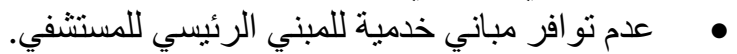

تو افر مساحات بالمبني الرئيسي للمستشفي غير الرئي مستغلة. الحاجه إلي تعديل المسقط الأفقي لغرف الإني الشعة و العمليات و الحضانات و ذللك لزيادة السعة و وجود تهالك بهم و غير مطابقة للمعايير التصميمية. تهاللك كل من سكن الأطباء و الممرضات و اللهونة الثؤون الماليه و الإدارية، و الحاجة إلي القيام بتعديل المسقط الافقي لتحقيق السعة المطلوبة. وجود مشاكل في أنظمة و شبكات الكهرباء و وجود ودود تهاللك بهر.

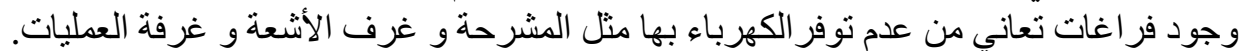

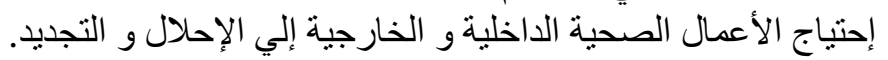

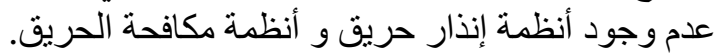

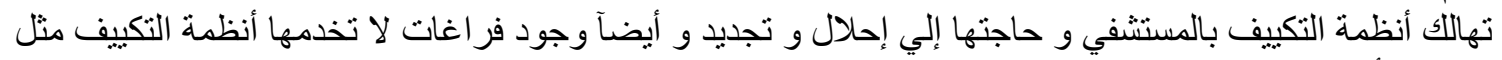
غرف الأشعة و المعمل و العمليات و الحضانات الحنات

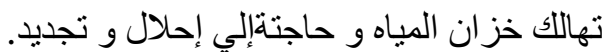

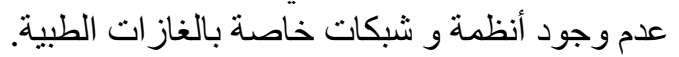
لا يو يد مصاعد كهربائية. و جود قصور في العلاقات الوظيفية للأدوار و غير مطابية للابة للمعايير و الإشتر اطات التصميمية. الحاجة إلي عمل سلالم خارجية منفصلة لكل دور.

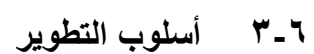

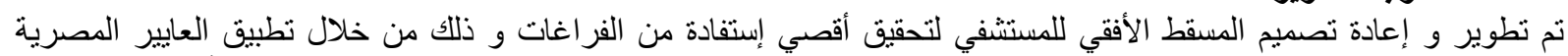

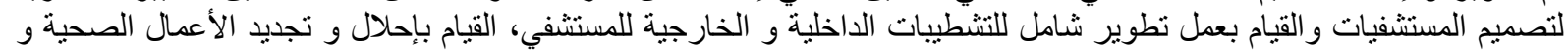
تعديل المسقط الأفقي لسكن الأطباء و الممرضات و والثئئون المالية و الإدارية و ذلك لتحقيق السعة المطلوبة.

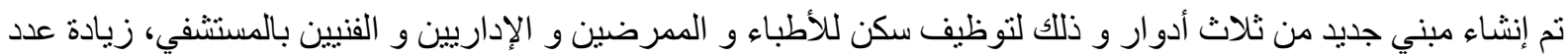

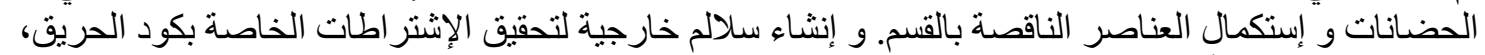

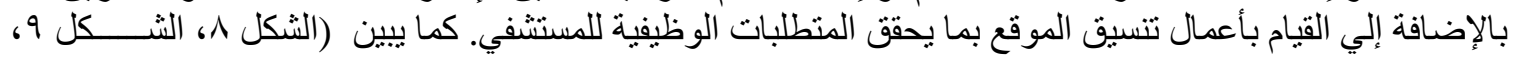
الثكل • (1) كروكي توضيحي لمكونات المستشفي بعد عملية التطوير. 


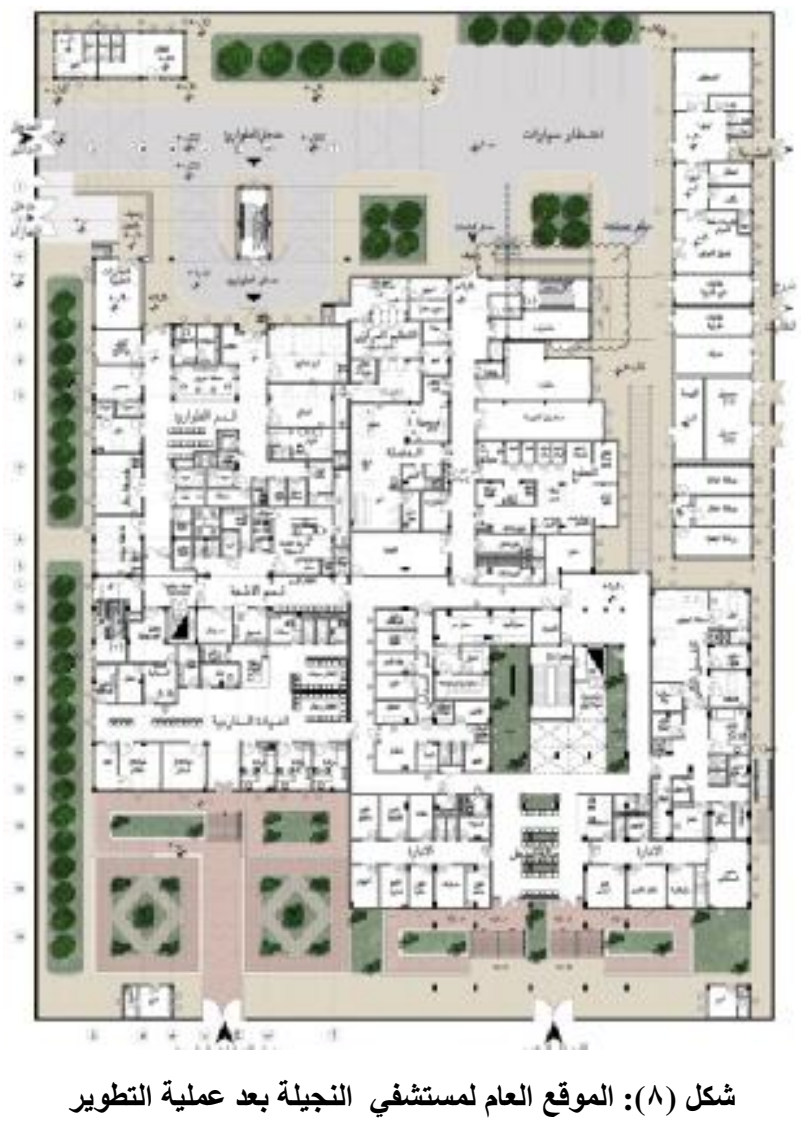

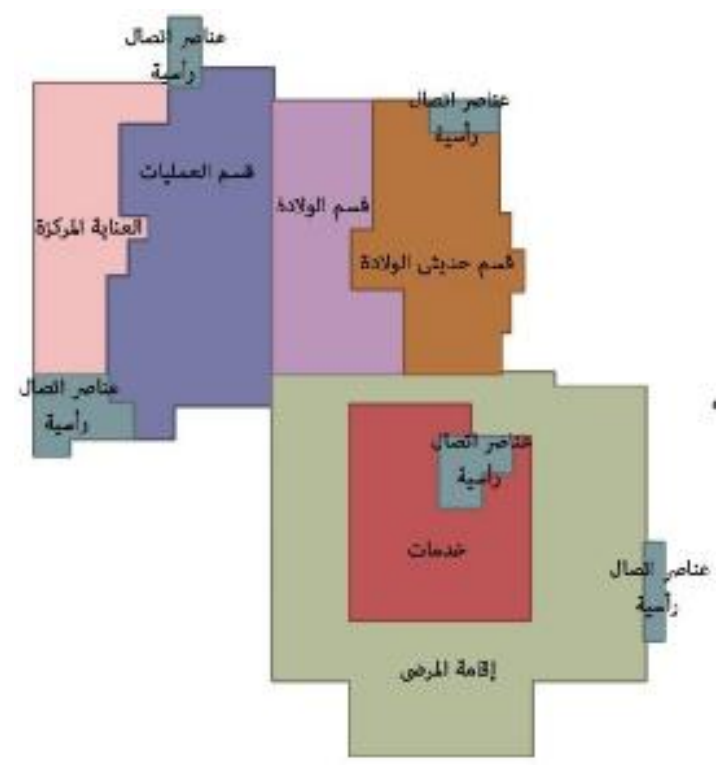

شكل (• (1): تحليل المسقط الأفقي للاور الأول بعد عملية التطوير

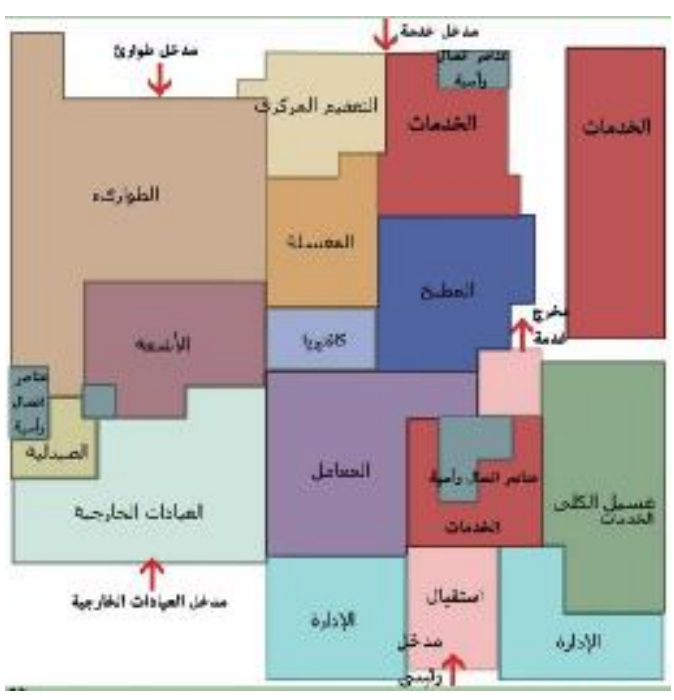

شكل (9): تحليل المسقط الأفقي للاور الأرضي بعد عملية التطوير 
ـ وجود نقص في الخدمات الطبية المقدمة من بعض المستشفيات و عدم كفاية الحاجة المجتمعية من تلك الخدمات

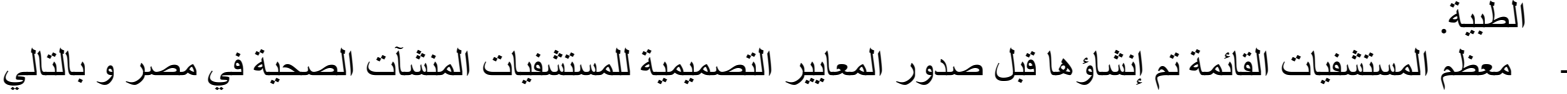
تللك المستشفيات لا تحقق المعايير و المنطلبات التصنيات التصميمية للمنشآت الصحية الصنية. - يمكن القيام بتعديل المستشفيات القائمة و تطوير ها من خلال تحديد إستر اتيجية للنطوير تتناسب مع الوضع الر اهن و تحقق الإحتياجات المستقبلية. تحتاج بعض الأحيات الأقسام إلي التطوير لكي تحقق التوازن بين العناصر الناقصة و ذلك في حالة وجود نقص في قسم قائم بالفعل. يتم اللجوء لعملية النطوير و زيادة حجم العناصر القائمة للوصول إلي توفير الخدمة الجيدة للمنطقة المحيطة لنطاق المستشفي؛ و ذلك لعدم كفاية و لا تو افر إحتباجات المنطقة المنات المحيطة. يتم اللجوء إلي رفع كفاءة و تحديث التجهيزات الفنية الموجودة في المستشفي لتقديم خدمة طبية جيدة من خلال تجديد الميدة الفرش و الأجهزة الطبية و شبكات الغازات الطبية و أنظمة التكييف و الصرف الكيكات الصحي و كل ما هو متعلق بأنظمة الكهروميكانيك. ـ ـ تعد عملية تطوير المستشفيات القائمة ذات أهمية قصوي للإستفادة بالموارد المتاحة في حالة إرتفاع تكلفة إنشاء

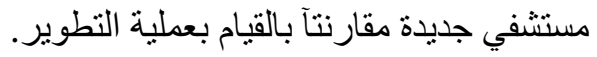

- مالت ضرورة الدراسة الجيدة للوضع الر اهن للمستشفيات القائمة و تقييم مدي حاجنها لعملية التطوير و تحسين الخدمة الطبية بها.

ـ ـ ـ يجب تطوير المستشفيات القائمة بما يتو افق مع المعايير و الإشتر اطات التصميمية للمنشآت الصحية المصرية.

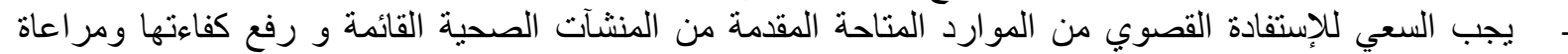

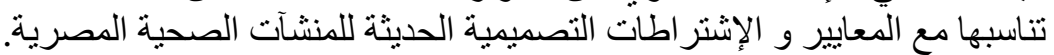

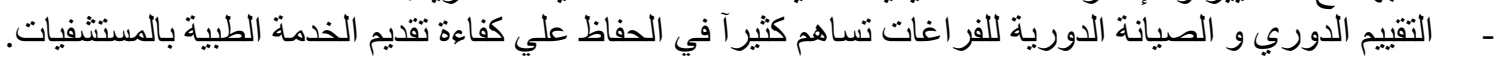

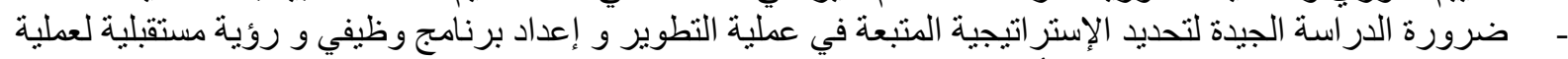
التطوير و ذللك لتلافي وجود معو قات أثناء عملية التطوير.

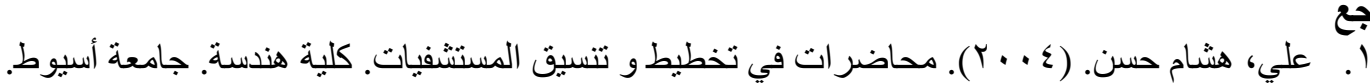

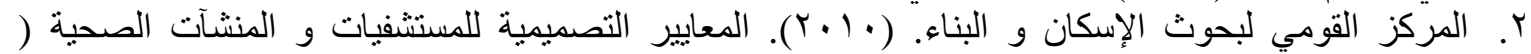

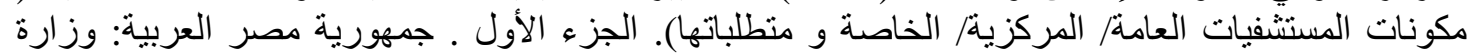
الإسكان و المر افق و التنمية العمر انية.

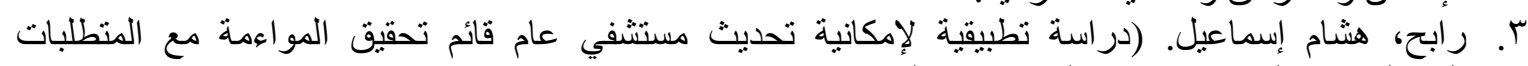

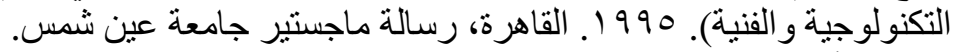

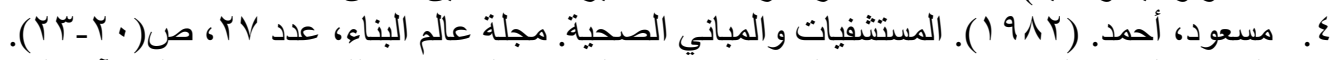

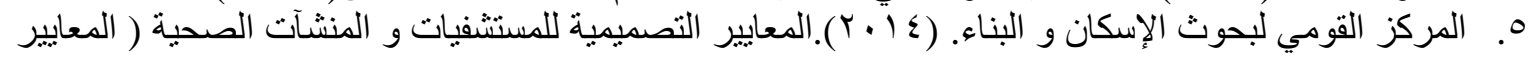

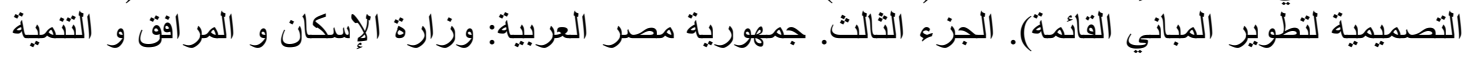
العمر انية.

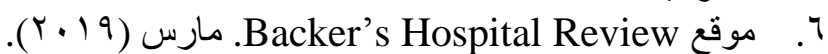
https://www.beckershospitalreview.com/strategic-planning/hospital-renovation-or- .V .new-construction-get-with-the-plan.html 